\title{
Good practices in harnessing social media for scholarly discourse, knowledge translation, and education
}

\author{
Daniel Lu (D) B Brandon Ruan (D) - Mark Lee (D) - Yusuf Yilmaz (D) Teresa M. Chan (D)
}

Published online: 20 August 2020

(C) The Author(s) 2020

\begin{abstract}
Introduction There still remains a gap between those who conduct science and those who engage in educating others about health sciences through various forms of social media. Few empirical studies have sought to define useful practices for engaging in social media for academic use in the health professions. Given the increasing importance of these platforms, we sought to define good practices and potential pitfalls with help of those respected for their work in this new field.

Methods We conducted a qualitative study, guided by constructivist grounded theory principles, of 17
\end{abstract}

Electronic supplementary material The online version of this article (https://doi.org/10.1007/s40037-020-00613-0) contains supplementary material, which is available to authorized users.

\section{Lu}

Department of Psychiatry, Faculty of Medicine, University of British Columbia, Vancouver, BC, Canada

B. Ruan · M. Lee · Y. Yilmaz · T. M. Chan $(\bowtie)$

McMaster Education Research, Innovation, and

Theory (MERIT) unit, McMaster University,

Hamilton, Ontario, Canada

teresa.chan@medportal.ca

\section{Y. Yilmaz}

Department of Medical Education, Faculty of Medicine, Ege University, Izmir, Turkey

\section{Y. Yilmaz · T. M. Chan}

Program for Faculty Development, Office of Continuing

Professional Development for the Faculty of Health

Sciences, McMaster University, Hamilton, Ontario, Canada

\section{T. M. Chan}

Department of Medicine, Division of Education

\& Innovation and Division of Emergency Medicine,

Faculty of Health Sciences, McMaster University,

Hamilton, Ontario, Canada emerging experts in the field of academic social media. We engaged in a snowball sampling technique and conducted a series of semi-structured interviews. The analytic team consisted of a diverse group of researchers with a range of experience in social media. Results Understanding the strengths of various platforms was deemed to be of critical importance across all the participants. Key to building online engagement were the following: 1) Culture-building strategies; 2) Tailoring the message; 3) Responsiveness; and 4) Heeding rules of online engagement. Several points of caution were noted within our participants' interviews. These were grouped into caveat emptor and the need for critical appraisal, and common pitfalls when broadcasting one's self.

Discussion Our participants were able to share a number of key practices that are central to developing and sharing educational content via social media. The findings from the study may guide future practitioners seeking to enter the space. These good practices support professionals for effective engagement and knowledge translation without being harmed.

Keywords Social media - Knowledge Translation · Education · Scholarly Discourse

\section{Introduction}

Social media is now a dominant medium for discourse, debate and education. Recently, the COVID19 pandemic has highlighted how crucial social media has become for both our daily and professional lives $[1,2]$. With the staggering explosion of content online both within health professions education and knowledge translation [3], we are entering into an attention economy for learners within the increasingly crowded social media space [4]. Within business, marketing professionals manage their brand's attention in 
a vast sea of attention-seeking stimuli. In the online world of health professions education, a similar attention economy is emerging; teachers and scientists are deploying many different tactics to engage their intended audiences.

One of the great challenges in engaging scientists and investigators (including those in health professions education) in social media activities is their perceptions of its usefulness. Some individuals who have spent their lives devoted to generating science may not feel adequately trained to engage in social mediabased techniques for disseminating their scholarship $[5,6]$ or sufficiently rewarded by traditional tenure and promotion processes [7]. These factors have contributed to social media's under-utilization by the scientific community despite it having shown to have direct implications for enhancing visibility of science $[8,9]$.

In a recent scoping review, while there was an abundance of descriptive studies $(n=242)$ and conceptual pieces $(n=192)$, there were very few clarification studies $(n=5)$ about the usage of social media for education and/or knowledge translation [3]. None of these studies attempted to define good practices used by experienced providers. We sought opinions from those respected for their academic social media work to generate a list of good practices and potential pitfalls.

\section{Methods}

We conducted a constructivist grounded theory study to determine the good practices and potential pitfalls observed by experts in the areas of social media education and knowledge translation.

Sampling We engaged in a snowball sampling technique, which has been used in the field of social media research within health professions education since it is an evolving field with rapidly changing techniques and protocols [6, 10-12]. We initially randomly drew from a previously published list of social media influencers within emergency medicine [12], since this field has been shown to be quite active in social media scholarship and publications, according to a recent review [2]. However, since there has been a marked adoption of social media across all sectors since this original list, we employed a snowball sampling technique as the expertise in this area is not fixed and is evolving. As such, snowball sampling allowed our interviewees to then further nominate individuals whom they admired as experts in one of the following areas: 1) Knowledge translation and teaching; 2) Acting as an interactive scientist or investigator; 3) Engaging as a critical clinician [5]. Individuals were initially contacted by email or social media to engage in our study. We attempted to sample across all three groups.
Context The context of the study was the digital community of social media knowledge translation specialists and educationalists. Although we began our study of social media experts using a social media influencers list from one specific specialty (emergency medicine), our context was broader due to our snowball sampling.

Ethics Our team received ethical approval from the Hamilton Integrated Research Ethics Board (\# HIREB5609).

Data collection methods A series of semi-structured interviews were conducted by our team's research assistants (BR, AM). The research assistants were initially trained via simulation and practice with feedback. Initial transcripts for research assistants were also reviewed initially by the primary investigator (TC) to provide insights for further topic exploration as part of our constant comparative analysis. Each interview was conducted using Zoom (Zoom Video Communications, Inc., San Jose, CA, USA) with audio capture on our local computer. The interview guide is found in the Electronic Supplemental Materials (Appendix 1).

Data processing The audio files were sent to a trained and experienced medical transcriptionist, who generated written transcripts from the audio files. Participants were assigned a gender-matched alias. The transcripts were then verified or corrected as needed by the interviewer and investigatory team to ensure the accuracy of the transcript.

Data analysis We conducted our analysis using a constant comparative method, iteratively delineating a series of codes aligned with various good practices and potential pitfalls throughout our coding process. Our analysis team (BR, DL, TC) met multiple times over a number of months, analyzing transcripts for relevant themes after batches of 2-4 interviews were completed. Each coding session, a code book was updated, with relevant codes being organized and reorganized until we reached thematic sufficiency within our dataset about good practices and potential pitfalls to avoid. These analysis sessions allowed us to iteratively refine the prompts or subprompts used by our interviewer, guiding us better towards sufficiency.

Sensitization In constructivist grounded theory, researchers may be sensitized by concepts that have preceded their present work. These are concepts that inform their analysis and are acknowledged fully for the readership. For our analysis we were sensitized by two concepts: 1) Davenport and Beck's attention economy [4]; and 2) The new types of social media scholars (translational teachers, interactive investigators, and critical clinicians), which was a conceptual 
framework that had been previously proposed in the literature [5].

The concept of the attention economy comes from a concept in the business world which highlights the increasing limitations of end-user (or customer) attention as a new type of economic driver; specifically, human attention is now a form of currency due to limitations in its supply, thereby forcing those who demand our attention to compete. Davenport and Beck's work was mainly used for the analysis portion of our study. When reading transcripts, this concept helped us to detect practices that were more akin to business structures or marketing strategies that our experts were employing for the purposes of disseminating education. Their work describes several key concepts within the attention economy: voluntary attention, attractive attention, aversive attention, frontof-mind attention, and back-of-mind attention. Moreover, they describe the concept of attention management, a task that seems to resonate with educators.

The other concept of the new types of social media scholars, which emerged due to the increasing use of social media for knowledge translation, has been highlighted because our principal investigator (TC) was a lead author on this conceptual work, and its influence is undeniable in this present study-we framed our recruitment and interview guide around these types of scholars, specifically seeking out those who fit within this framework.

\section{Techniques to enhance rigor and trustworthiness}

To ensure rigor of our analysis, we engaged two members of our research team (ML, YY) to conduct an audit of our analysis trail. They were given full access to primary transcripts and the final codebook. The plan for resolving conflicts at this stage was to engage in discussions around areas of concerns. Consensus building techniques were used to resolve any issues that arose.

Reporting This report adheres to the Standards for Reporting Qualitative Research reporting guidelines [13].

\begin{tabular}{|c|c|c|}
\hline Platform & Best practices & Examples mentioned \\
\hline \multirow[t]{5}{*}{ Twitter } & \multirow{2}{*}{$\begin{array}{l}\text { Users may find it prudent to divide out different } \\
\text { accounts for different usages. Some suggested } \\
\text { divisions: } \\
\text { - Person-level professional account } \\
\text { - Group/Institutional } \\
\text { - Research Team }\end{array}$} & $\begin{array}{l}\text { Group/Institution: } \\
\text { @WeAreCanadiEM (www.canadiem.org) } \\
\text { Residency Program account } \\
\text { Departmental account }\end{array}$ \\
\hline & & $\begin{array}{l}\text { Research Team: } \\
\text { @METRIQstudy (www.metriqstudy.org) }\end{array}$ \\
\hline & \multirow{3}{*}{$\begin{array}{l}\text { When engaging in social media promotion of } \\
\text { research, consider the following practices when } \\
\text { generating a tweet: } \\
\text { - Include an image in the tweet } \\
\text { - Use descriptive language } \\
\text { - Tag people involved } \\
\text { - Tagging related organizations or granting agencies } \\
\text { involved in the work } \\
\text { - Tagging the journal that the article was published } \\
\text { within } \\
\text { - Using hashtags to join the right conversation } \\
\text { Advanced concepts include: } \\
\text { - Tweet chats }\end{array}$} & $\begin{array}{l}\text { Understand the nuances between accounts. Have a clear intent and purpose for each } \\
\text { account }\end{array}$ \\
\hline & & $\begin{array}{l}\text { "You have to be aware of what the purpose of each account is and certainly the } \\
\text { purpose of my department's account is very different than my account."-Piper }\end{array}$ \\
\hline & & $\begin{array}{l}\text { "I am really deliberate in my use of hashtags. I also try not to spam. (Laughing). } \\
\text { So, like three or less hashtags in a tweet... Also, in my communication, I will } \\
\text { take tag certain people that I want to make sure that they are aware."-Grace }\end{array}$ \\
\hline \multirow[t]{2}{*}{ Facebook } & Person-level account & N/A \\
\hline & Facebook pages-Group/Institutional & CanadiEM Facebook Page \\
\hline Instagram & Group/Institutional & $\begin{array}{l}\text { PEM Morsels } \\
\text { CanadiEM }\end{array}$ \\
\hline $\begin{array}{l}\text { Closed social } \\
\text { platform }\end{array}$ & $\begin{array}{l}\text { Used for within team communication to enhance } \\
\text { the functioning of a team of social media users or } \\
\text { producers (e.g. blog community) }\end{array}$ & $\begin{array}{l}\text { Groups using Slack: } \\
\text { ALiEM } \\
\text { CanadiEM }\end{array}$ \\
\hline Blog & $\begin{array}{l}\text { Used for housing general summaries and dissemina- } \\
\text { tive works, but also to release new scholarly contribu- } \\
\text { tions via a digital platform. }\end{array}$ & $\begin{array}{l}\text { "... we have been producing a case of the week. And disseminating that internation- } \\
\text { ally with our pathology residents and fellows ... using blogging platform to do that } \\
\text { with the question. It is a short snippet of the case-80 words or less. It has an im- } \\
\text { age or digital image. Like a digital scan and pathology slide as well as the question } \\
\text { that goes with it. So that is another way that we have used social media for learners } \\
\text { and also for our faculty."-Grace }\end{array}$ \\
\hline Podcasts & $\begin{array}{l}\text { A possible outlet for digital scholarship and academic } \\
\text { output. Can be used as its own free-standing aca- } \\
\text { demic output, since it is seen as digital scholarship }\end{array}$ & $\begin{array}{l}\text { EM Basic (for junior trainees) } \\
\text { EM Guidewire (involves residents) }\end{array}$ \\
\hline
\end{tabular}


Table 2 Good practices for engagement online

\section{Good practice \\ Explanatory quote}

Use common sense

Clearly identifying yourself, including

Aligned with self and institution

Understand the intention of each account in each platform

Maintaining respect

Avoid arguments

Knowing when to end a conversation Anticipate trolls

Amplify others this."-Paula conflicts of interest I am responding to somebody it will usually be to make sure that I say something positive or say nothing at all."-Sheila

"I clearly identify myself as my Twitter handle is not my name, but my name is on my Twitter profile. And yeah, I think that is pretty straightforward. .. like, just behave properly."-Sheila

"It's just really thinking about your profile is a best practice. Just thinking about being transparent to the community [about] who you are and ... what you are going to be communicating about in that social media platform. So, [regarding] your presence in your profile, I think another best practice that I really try and think about and encourage other people to think about as well."-Grace

"I've tried to make all of my intentions honorable and things that I would be proud of representing and that would reflect on my institution and institutions in a positive way. And so, my interactions again are founded on what is going to be best for patient care and kindness and making my intentions honorable. And so those are all things that I think of as core values that the institutions that I am affiliated with ... support."-Edward

"I am a big believer in aligning my technology with my goals that I want to achieve. And also separating personal and professional. So I chose Twitter because at the time it was where I was connecting with people in medical education, finding that it seems like that is where the audience that I wanted to connect with professionally was currently at. I felt like Facebook was more personal. Um, and that Instagram and other, and Instagram especially I guess was just starting to emerge when I was working with getting myself established in medical education. Um, now have I moved to Instagram. I use Instagram, um, more to help I guess personal[Iy], but I guess some I had done some work connecting with other professionals on it just a little. Slack is one that I use ... "-Grace

"I think in general you try to um, be polite and professional. Like I don't necessarily think delving into in depth articles on Twitter is necessary or appropriate, um, however responding to people who are having questions or being critical of things I think it is a very reasonable way to go. And [l] try to do it in a respectful way... And that can be productive [in] conversation"-Trevor

"... always assume that if there is two ways to read something then thinking the kinder way is the way that somebody wants you to read it; I think it is a good rule of thumb because you know like I said, it is hard to interpret tone."-Anthony

"So, [an important aspect is] being respectful, you know only saying things that you would say to other people for the most part being particularly I would say from a department account you know being very positive about all of the people that you work with. I think kind of from a formal account, really you probably have to be positive, [an] uplifting voice."-Piper

"I am always surprised at how argumentative some people get. And I think that is a little bit of a shame because I don't think that ... sort of reflects well and this idea about somewhere in between you know maintaining some appropriate composure versus being a skeptic and questioning things. And there are definitely some people who do a good job of that and some people that don't."-Sheila

"If there are people that are engaging that seem to have a substantial agenda, then I am more likely to not continue the conversation for long while still being respectful and just [stop] interacting." -Trevor

"I mean you will occasionally get trolled by negative people...I thankfully haven't had too much with that but every once in a while, something that I put it out on \#1, some naysayer will put something negative or sort of like oh it is just like \#6 to do something like

"You know there [are] always trolls, right? But I think of one, so before I hit publish on anything, I am like super critical of myself first. So, I think if you already are highly concerned about the words that you use and the product that you are publishing then you are going to find that most people are not out there to be obstinate and/or aggressively negative. And if there is a question then usually it is raised with a more honest and um, straightforward inquiry rather than being malicious." - Harold

Engage across silos "I tried to actively engage others across multiple specialties and disciplines. So not just emergency physicians but other physicians, and not just physicians but nurses and technologists and the public. So, it is mostly I think the fact that I tried to cross barriers that might otherwise limit the scope of other people who are on social media."-Roger

"if there was someone that I know that is doing something cool or having something awesome to happen then I might favorite that or retweet that."-Trevor

"I tried to disseminate most of the work that we publish. I try to, anything that we publish that I think is worth making people aware of, I will put a plug in for it. Sometimes I will do a Twitter thread if it is a particularly important study. And I will often tag junior investigators or colleagues to increase their follower count."-Nadir

\section{Results}

\section{Demographics of participants}

In total, 17 individuals were interviewed. See Tab. 1 of the online Supplementary Material for details about key demographics. Social media platforms used by our participants can be found in Tab. 2 of the online Supplementary Materials, which is also online. The interviews were on average $30.6 \mathrm{~min}$ long, ranging from 18.6 to $52.1 \mathrm{~min}$. This yielded a total of 189 pages of transcripts. Within this group, nine individuals self-identified as translational teachers, five indi- viduals identified as critical clinicians, and three saw themselves as interactive investigators.

\section{Key themes}

Overall, there were several good practices that were felt to be important. Specifically, the domains that our good practice tenets fell within included: 1) Understanding the nuances of specific platforms; 2) Social media team management; 3) Online engagement strategies; 4) Techniques of effective knowledge sharing; 5) e-Professionalism; 6) Potential pitfalls. The following sections detail the perspectives of the various 
participants, who are named by their randomly selected aliases.

\section{1) Understanding the nuances of specific platforms}

One aspect of good practices was knowing and harnessing the specific platforms available within the social media space for effective engagement. Understanding the strengths of various platforms was deemed to be of critical importance across all the participants. Tab. 1 depicts some key platforms that were mentioned and clarifying examples are provided when possible. One participant (Edward) put it well when he described that each of the various media have their own temporal properties: "They each play a different role... It depends on what the ultimate goal of the interaction is. Certainly, Twitter has much more frequent interaction. The blog is, you know, a weekly thing as is the podcast and then YouTube might be a monthly thing".

\section{2) Social media team management}

Although some participants retained single-person access to social media accounts (usually around their personal accounts), the preferred mode of conduct for group or institutional social media accounts was to enable shared access across multiple users. The rationale for this was that shared access connoted shared accountability, which made the work lighter for any one person. This finding was also true for blogs and podcasts. Although some of our participants still engaged in single-person blogging and podcasting, many have involved bigger teams. Some even saw these as opportunities to engage in teaching trainees. Harold, for instance, involves residents in his process:“ ... [M]y residents ... come up with topics ... So, that is much more of a group collaboration fashion and we will ... edit the script and figure out you know what the teaching points should be..."

\section{3) Online engagement strategies}

We found a number of key engagement strategies that were mentioned by our participants as crucial for building online engagement: culture setting strategies, tailoring the message, responding and responsiveness, and heeding rules of online engagement.

Culture setting strategies Some of the key engagement strategies mentioned by the group were tied to specific platforms or tactics, but others were more generic. Generally speaking, some participants thought that creating an open, welcoming environment was crucial to engagement. One participant, Grace, stated:

I try and really welcome people. And make sure that when engaging them and there is somebody new in a social media environment, um, synchronous or asynchronous discussion, I make sure to welcome that person ... I try and think about netiquette [sic]. And helping people feel successful when they are using social media...

For some, creating a culture also meant monitoring the quality of online discussions (especially ones they were engaging within) and getting involved when necessary to halt or modify conversations, or to actively avoid frank arguments. For others, this means setting a positive tone with the hopes of actively creating a productive space for sharing. One participant (Piper) noted that “... from [an institutional] account really you probably have to be positive, [an] uplifting voice".

Finally, one other act of culture building identified by our participants was the need to teach and mentor others in this space. Culture building was thought to be collaborative, by encouraging faculty and trainees to engage together via social media for education. Some participants used their podcast or blog as a platform for engaging trainees, apprenticing them into this world while creating new content.

Tailoring the message Our participants thought that social media messages should be tailored (language level, style) to the audience, as Nadir states:

I have a sense of who I want to read it. And so, if I want the general public to read it then I minimize the jargon and I make it sort of you know interesting to people who are non-medical. But if I want people in my specialty to read it then I don't mind getting extremely technical.

Responding and responsiveness Other keys to engagement were thought to be around being responsive to others. From one participant's point of view (Trevor) it was crucial as an investigator on Twitter to interact with those who sought you out. He stated: "I don't necessarily think delving into in-depth articles on Twitter is necessary or appropriate... however, responding to people who are having questions or being critical of things I think is a very reasonable way to go". Meanwhile on the receiving end of such engagement, others certainly found that this type of interaction was helpful to themselves as scientists as well.

Responsiveness was thought to have its dark side as well. As you engaged more, our participants highlighted the need to know when to stop having a conversation too. For some, it simply meant halting engagement. For others, they thought it was important to think twice before responding. Taking the emotion out of a disagreement was one strategy highlighted by one of our participants (Jason). He highlights his own strategy for dealing with disagreements online:

I tend to not respond emotively [sic] to anything. If I like something, I will like it. Or I may put a fairly neutral response like: "we have done something similar have a look at this paper". Rather than 
saying "you know you're wrong and we are right for these reasons..."

Heeding rules of online engagement Many of the other keys to interaction revolved around some rules on using common sense to engage in respectful and positive conversations, while also understanding your goals/intentions for communicating. These rules for engagement are summarized in Tab. 2, alongside explanatory quotes.

\section{4) Techniques for effective knowledge sharing}

Participants made note that creating a digital home base such as a website was thought to be of great importance, but largely this was thought to be insufficient for effective knowledge sharing. Social media sharing of new research was thought to be best if it was multimodal, in order to be most useful in promoting that knowledge to the end-users. But simply creating a website and sharing was not thought to be sufficient. There was a perceived need to 'repackage' content in a way that was engaging within a specific medium. Bearing in mind the audiences in the social media space, participants like Anthony noted that the role of a good teacher or translationalist in this space is to "... make [core concepts] easily understandable, accessible, you know put my own little spin and little pearls...".

Julie noted that while repackaging was of importance, it was crucial to be knowledgeable about endusers. She said that for her podcast, she and her cohost try their best to bear in mind their listenership. She stated:

\section{... we try to make sure that we explain sort of the basics instead of just assuming because we know we have a lot of medical student listeners and a lot of early resident listeners, in addition to career emergency physicians. And so, we've tried to cover the gamut by covering stuff that is interesting to us as ... docs but then also to go into a little bit of the sort of basics to an extent, to kind of address the [other audience groups].}

Some participants also highlighted the need to both produce and selectively amplify high-quality, accurate content. This responsibility was best stated by Anthony who said how crucial it is for producers to be "just making sure that whatever content you put out, that its quality is accurate, is important as well". In an effort to ensure the quality of any educational or knowledge translation projects they undertake, some individuals create networks of trusted advisors and reviewers to review their work prior to publication, an ad hoc version of pre-publication peer review, which may or may not be subjected to further scrutiny by editorial members of a social media outlet. Piper stated: “... if it is at all anything controversial, I usually send it along to a trusted mentor or a friend to read and give me their thoughts on. And then it goes to the editorial board".

Sharing one's own research Good practices for disseminating one's own research included ensuring that various social media promotional activities were all aligned with the area of research or scholarly interests. Participants highlighted that it is useful to consider integrating alternative media into the knowledge translation process. Specifically, infographics were thought to be of importance in this area, either as a post-publication dissemination technique or directly into research papers so as to facilitate social sharing of the paper by others. Creating a planned and integrated strategy for disseminating a paper after it is published can include alternative and creative forms of expression including infographics, as Piper vividly describes:

... the other kind of variety of content that I seem to be producing most of these days is like infographics for translation of our research findings... [infographic creation] requires you to do is really distil down what this big study is about ... really, it gets you thinking: 'what is the impact of what we have done?'... '[H]ow do I want to share and frame that for people?' So, when I do those it ends up being a bit of a self-reflective process on my paper or my research and what it is bringing to the community.

The challenges of infographics of course are that the visual medium requires a different type of thinking and careful design is important so as not to over-simplify or water down the content. Julie stated: “... for creating the visual abstracts, I create those for the lay emergency clinician who does not understand large relative risks or odds ratios ... And then I tried to convey the results in an as succinct ... way that I can".

\section{5) e-Professionalism}

Similarly, for those who inhabit the online space more as 'translationalists' and teachers, the space is similarly riddled with traps. As stated previously, it is imperative for professionals (e.g. physicians and nurses) engaging in online education to be wary of their professional obligations, and all individuals should be aware of how easy it is to unintentionally breach confidentiality. As Harold points out, training in this area is a must: "We, you know, are very cautious with our residents and faculty and making sure that we are training everybody that you know you are not publishing things that are sensitive material or patient information and all of the common pitfalls that we have seen". And even then, educators in the online space should be continually vigilant in assisting those new to the field since, Brandon remarked, “... there is, you know, potential for ... even unintentional patient privacy violations ... which again can get people into trouble with their home institutions". 


\section{6) Potential pitfalls}

Several notes of caution were noted within our participant's interviews, which we grouped into two broad categories: 1) Caveat emptor and the need for criticality with resources; 2) Common pitfalls when broadcasting one's self.

Caveat emptor: The need for increased rigor and criticality for social media resources Specifically, some participants felt that it was important for users of social media resources to note that their work was not a comprehensive resource, and that they (as the sellers of the evidence) could not replace primary literature or textbooks. Participants highlighted the value of social media to curate or highlight important resources, while also recognizing that sometimes social media will highlight sensationalist (and less rigorous) resources at times. Trevor noted: “I don't believe that the use of social media replaces any of my academic reading". Specifically, Trevor noted that it was important to make clear to users that social media-based resources should not supplant the use of the primary scientific literature. This was similarly mirrored by other comments who cautioned those in this space to recognize their responsibility for fact-checking and ensuring accuracy of online content prior to distributing it.

Common pitfalls when broadcasting one's self When engaging in disseminating one's own work on social media, one must be aware that others may not perceive this as a simple act of sharing. As one participant (Piper) highlighted, there was a fine line that needed to be walked between 'bragging' and disseminating your content. This quote highlights her perspective:
You know I think the biggest balance is... towing this line between self-promotion and sharing your resource or sharing your research and getting stories out about your studies and about what you think is important... I don't want to be seen as pushing it at people aggressively... I also want to be seen as humble and thoughtful.

Many of our participants felt the weight of responsibility upon them when speaking about their role as an openly identifiable online physician. Examine this one statement by a participant (Darcy):

... [T]here is a ... reason that the Doctor Oz show is the Doctor Oz [show], and it is not just [titled] talking about some shit [sic] with a guy named Bennett. I mean, there is gravitas. There is a professionalism to being a physician.... [there], is a factor of credibility because anything that I put out there I sign my name to.

\section{Discussion}

In this study we have sought the insights of peeridentified influencers and leaders within the social media learning environment to understand good practices and potential pitfalls for those entering this space. Through their aggregate experiences, several key themes are summarized in Fig. 1 as key takeaways for our readers.

Our findings should be interesting to both new scholars, who are seeking to carve their niche within the academy, but also those seeking to foster others' success by capitalizing on social media as a platform for dissemination. There is an evolving role for new scholars in today's academic milieu who can help
Fig. 1 A summary of our study's themes around key considerations in the use of academic social media

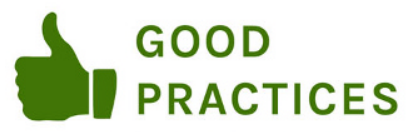

Understand the nuances of specific social media platforms.

Consider taking a team approach

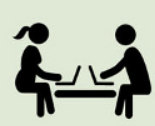
to manage the social media presence of an organization.

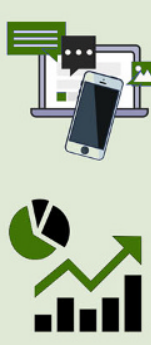

Use varied online engagement strategies ensures that stakeholders feel welcomed to participate.

Use evidence-informed techniques for effective knowledge sharing wherever possible.

\section{g priaul AVOIDANCE}

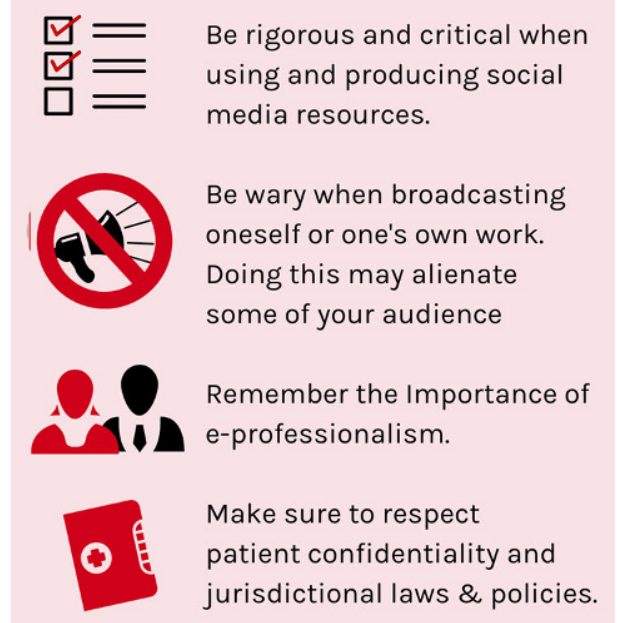


with the translation of knowledge as teachers (translational teachers), and those who effectively engage their target audiences and key stakeholders via social media as scientific investigators (interactive investigators) [5]. This paper provides empirical data which highlight these new roles, and how they are increasingly sophisticated. Participants who identify with these roles have a growing mandate to organize and add structure to a zone where social media meets academia. This aligns with the structuralist phase of the greater Free Open Access Medical education (FOAM) movement [14], which includes new roles such as Social Media editors for journals [15].

Our participants also explained how they are not solely translational teachers, but at times must wade into promoting their own scientific or scholarly work, which shifts their role and requires new considerations. As critical clinicians, they saw the need to be actively skeptical of the science and participate in scholarly discourse around science that is published in any format-whether it be in a high-impact journal or a high-traffic blog. As teachers, our participants reflected upon how they seek to produce high-quality content and to educate others to appraise content in the social media space. Finally, as either interactive investigators or translational teachers, they remarked on their sense of responsibility around the need to be accurate and not fall into the trap of becoming a 'celebrity' or 'science Kardashian' [16-18]. Due to their professional identity, our participants found it imperative to consider content accuracy and saw themselves as accountable for ensuring the validity and veracity of their content. Similarly, e-professionalism was found as a thread throughout the interviews; the themes found in our present study were similar to work that has been done on e-professionalism within medical student and trainee populations [19-22]. That said, compared with prior literature [19, 22] which largely focused on the hazards of social media towards professionalism, our participants heavily de-emphasized this concept, relegating it to a concept that must be incorporated but not in the front of their minds.

Many of our findings show the parallel between our participants' social media use and the techniques used by modern marketing strategists to gain attention; for example, repackaging content in various formats to suit consumers (e.g. infographics, podcasts, easy-to-read blogposts) and adequate brand alignment between content producers and target audiences were identified as essential practices. Many of the concepts discussed by Davenport and Beck [4] have a suitable mapping to the online engagement strategies.

As Davenport and Beck write in their book, those that: “... succeed in the future will be those experts not in the time management, but in the attention management" [4]. Phenomena such as infographics and visual abstracts are tightly associated with tac- tics that would help grab learners' attention and prevent the TL;DR ("too long; didn't read") label that is dreaded in the social media world [23].

Translating longer articles to capture attention is a phenomenon that aligns very closely to the existence of an attention economy within social mediabased knowledge translation and medical education. Tailoring the message to your target audience helps you to capture voluntary attention through the effective structuring and design of your content. Being responsive and responding well to others also help our participants to engage the attractive attention-reinforcing and providing positive feedback to those who engage with your material. Heeding rules of online engagement to avoid unwarranted negative reactions for violating the cultural norms is closely connected to the concept of avoiding aversive attention. Our participants also found it harder to walk the fine line between self-promotion and knowledge translation/dissemination, worrying about how their peers in the profession might view them. Many health professions education researchers and scholars may find that this resonates with them as well. Meanwhile, some types of attention that Davenport and Beck identify in their work go beyond the depths of what we found currently within the responses of our respondents. For example, our participants did not specifically speak to the value of creating an academic brand to capture the back-of-mind attention that Davenport and Beck describe; however, there is increasing discussion around this in academic medicine [24].

Our present study has a number of limitations. First off, our lead investigator is fairly immersed in the world of online education and knowledge translation, and this may have affected our interpretation of the participants' words. To optimize her distance from the actual respondents, we ensured that she did not interview any of the participants. We also involved the research assistants and transcriptionist in redacting the individual transcripts to ensure that she was not privy to the identity of the various participants. In the analysis phase, we used multiple strategies to ensure the rigor of our analysis, acknowledging that our lead investigator brought with her both her 'insider' expertise, affording us a unique perspective on these topics.

\section{The future of \#MedEd in social media}

Going forward, the evolution of thinking within the social media-based education space will likely become increasingly aligned with the thinking of the participants within our study. We foresee issues around ensuring that we grab the attention (as well as the hearts and minds) of our audiences in social media; this will be of growing importance going forward. With the rise of a generation of physicians who essentially grew up with social media, we will gradually see the integration of these platforms into 
our scientific and educational circles [25, 26]. And while we cannot generalize across a whole generation [27], it is clear that the global increase in usage and popularity of social media as a major communication platform provides concrete evidence of the changes in the way we communicate to learners and colleagues $[1,2,28]$. The depth of considerations and thinking on the various topics around safe social media utilization was coupled with an ease with which some of the participants understood how to best harness the power of these new media.

\section{Conclusions}

By engaging leaders and early adopters of social media as a tool for scientific discourse, knowledge translation and education, our work identifies good practices to guide health professionals and other stakeholders in a space that is rapidly growing in both pervasiveness as well as importance. Key strategies revolved around content delivery, audience engagement, and e-professionalism as well as being critical of one's own accuracy and role on social media.

Acknowledgements We would like to thank Aisha Mohamed, Emma Bridgwater and Priya Thomas for their assistance in the development and early recruitment phases of this study. We also thank Elizabeth Clow for her services as a transcriptionist for our project.

Funding Dr. Chan reports receiving funding from the PSI Foundation for this work via the 2018 PSI foundation Graham Farharquason Knowledge Translation Grant Recipient. Dr. Yilmaz is the recipient of the TUBITAKPostdoctoral Fellowship grant.

Open Access This article is licensed under a Creative Commons Attribution 4.0 International License, which permits use, sharing, adaptation, distribution and reproduction in any medium or format, as long as you give appropriate credit to the original author(s) and the source, provide a link to the Creative Commons licence, and indicate if changes were made. The images or other third party material in this article are included in the article's Creative Commons licence, unless indicated otherwise in a credit line to the material. If material is not included in the article's Creative Commons licence and your intended use is not permitted by statutory regulation or exceeds the permitted use, you will need to obtain permission directly from the copyright holder. To view a copy of this licence, visit http://creativecommons.org/licenses/by/4.0/.

\section{References}

1. Merchant RM, Lurie N. Social media and emergency preparedness in response to novel Coronavirus. JAMA. 2020; https://doi.org/10.1001/jama.2020.4469. Published online March 23

2. Gottlieb M, Dyer S. Information and disinformation: social media in the COVID-19 crisis. Acad Emerg Med. 2020;27:640-1.

3. Chan TM, Dzara K, Dimeo SP, Bhalerao A, Maggio LA. Social media in knowledge translation and education for physicians and trainees: a scoping review. Perspect Med Educ. 2020;9(1):20-30.
4. Davenport TH, Beck JC. The attention economy: understanding the new currency of business. : Harvard Business SchoolPress; 2001.

5. Chan T, Trueger NS, Roland D, Thoma B. Evidence-based medicine in the era of social media: Scholarly engagement through participation and online interaction. Can J Emerg Med. 2018;20(1):3-8.

6. Collins K, Shiffman D, RockJ. How are scientists using social media in the workplace? PLoS ONE. 2016;11(10):1-10.

7. Cameron CB, Nair V, Varma M, Adams M, Jhaveri KD, Sparks MA. Does academic blogging enhance promotion and tenure? A survey of US and Canadian medicine and pediatric department chairs. JMIR MedEduc. 2016;2(1):e10.

8. Chan TM, Bhalerao A, Thoma B, Trueger NS, Grock A. Thinking critically about appraisingFOAM. Aem Educ Train 2019 May 23;3(4):398-402. https://doi.org/10.1002/aet2. 10352.

9. Eysenbach G. Can tweets predict citations? Metrics of social impact based on Twitter and correlation with traditional metrics of scientific impact. J Med Internet Res. 2011;13(4):e123.

10. Cadogan M, Thoma B, Chan TM, Lin M. Free Open Access Meducation (FOAM): the rise of emergency medicine and critical care blogs and podcasts (2002-2013). Emerg Med J. 2014;31(e1):e76-e7.

11. Lagu T, Kaufman EJ, Asch D, Armstrong K. Content of weblogs written by health professionals. J Gen Intern Med. 2008;23(10):1642-6.

12. Riddell J, Brown A, Kovic I, Jauregui J. Who are the most influential emergency medicine physicians on twitter? West J Emerg Med. 2017; https://doi.org/10.5811/westjem.2016. 11.31299

13. O'Brien BC, Harris IB, Beckman TJ, Reed DA, Cook DA. Standards for reporting qualitative research: a synthesis of recommendations. Acad Med. 2014;89(9):1245-51.

14. Chan TM, Stehman C, Gottlieb M, Thoma B. A short history of free Open Access medical education. The past, present, and future. ATS Scholar. 2020; https://doi.org/10.34197/ ats-scholar.2020-0014PS.

15. Lopez M, Chan TM, Thoma B, Arora VM, Trueger NS. The social media editor at medical journals. Acad Med. 2019;94(5):701-7.

16. HallN.TheKardashianindex: ameasure ofdiscrepantsocial media profile for scientists. Genome Biol. 2015;15:424.

17. Cameron P, Carley S, Weingart S, Atkinson P. CJEM Debate Series: \#SocialMedia—social media has created emergency medicine celebrities who now influence practice more than published evidence. CJEM. 2017;19(06):471-4.

18. Khan MS, Shahadat A, Khan SU, et al. The Kardashian index of cardiologists. J Am Coll Cardiol. 2020;2(2):2019-21. Case Rep.

19. Cheston CC, Flickinger TE, Chisolm MS. Social media use in medical education: a systematic review. Acad Med J Assoc Am Med Coll. 2013;88(6):893-901.

20. DeCamp M, Koenig TW, Chisolm MS. Social media and physicians' online identity crisis. JAMA. 2013;310(6):581-2.

21. McCartney M. How much of a social media profile can doctors have? BMJ.2012;344:e440.

22. Roy D, Taylor J, Cheston CC, Flickinger TE, Chisolm MS. Social media: portrait of an emerging tool in medical education. Acad Psychiatry. 2016;40(1):136-40.

23. Heinzman A. What does "TLDR" mean, and how do you use it?. https://www.howtogeek.com/435266/what-doestldr-mean-and-how-do-you-use-it/. Accessed 6 June 2020.

24. Borman-Shoap E, Li STT, Clair StNE, Rosenbluth G, Pitt S, Pitt MB. Knowing your personal brand: What 
academics Can learn from marketing 101. Acad Med. 2019;94(9):1293-8.

25. Borges NJ, Manuel RS, Elam CL, Jones BJ. Comparing millennial and generation X medical students at one medical school. Acad Med J Assoc Am Med Coll. 2006;81(6):571-6.

26. Howell LP, Joad JP, Callahan E, Servis G, Bonham AC. Generational forecasting in academic medicine: a unique method of planning for success in the next two decades. Acad Med J AssocAm Med Coll. 2009;84(8):985-93.
27. Jauregui J, Watsjold B, Welsh L, Ilgen JS, Robins L. Generational "othering": the myth of the millennial learner. Med Educ. 2020;54(1):60-5.

28. RochwergB, ParkeR, MurthyS, etal. MisinformationDuring the Coronavirus Disease 2019 Outbreak: How Knowledge Emerges From Noise. Crit Care Explor Soc Crit Care Med J. 2020;2(4):e98. 